\title{
Anti-mitochondrial M2 Antibodies Enhance the Risk of Supraventricular Arrhythmias in Patients with Elevated Hepatobiliary Enzyme Levels
}

\author{
Hiroki Konishi ${ }^{1}$, Koji Fukuzawa ${ }^{1,2}$, Shumpei Mori ${ }^{1}$, Seimi Satomi-Kobayashi ${ }^{1}$, \\ Kunihiko Kiuchi ${ }^{1,2}$, Atsushi Suzuki ${ }^{1}$, Yoshihiko Yano ${ }^{3}$, Akihiro Yoshida ${ }^{1}$ and Ken-ichi Hirata ${ }^{1,2}$
}

\begin{abstract}
Objective Supraventricular arrhythmias are commonly detected in patients with anti-mitochondrial antibody M2 (AMA-M2)-associated myopathy. However, the prevalence of supraventricular arrhythmias in unselected AMA-M2-positive patients and the impact of AMA-M2 on supraventricular arrhythmias have yet to be fully investigated.

Methods We analyzed 384 patients (116 men; age, 60 [48-69] years), who underwent AMA-M2 testing following the detection of elevated hepatobiliary enzymes. Supraventricular arrhythmias involving atrial fibrillation, atrial flutter, atrial tachycardia, sick sinus syndrome, and atrial standstill were confirmed by a 12-lead electrocardiogram, 24-hour ambulatory monitoring, and physician-assigned diagnoses within the three years before and two years after the AMA-M2 test.

Results Seventy-seven (20\%) patients were positive for AMA-M2. The prevalence of supraventricular arrhythmias among AMA-M2-positive patients was higher than that among AMA-M2-negative patients (14\% vs. $6 \%, \mathrm{p}=0.008$ ). A univariate analysis showed that supraventricular arrhythmias were associated with AMAM2 positivity, aging, congestive heart failure, and the $\mathrm{CHADS}_{2}$ score. The multivariate analysis determined that AMA-M2 positivity was an independent risk factor for supraventricular arrhythmias (odds ratio $3.52, \mathrm{p}=$ 0.011). Among the AMA-M2-positive patients, the AMA-M2 titer did not differ to a statistically significant extent, regardless of the presence or absence of supraventricular arrhythmias. Multiple supraventricular arrhythmias with extremely low atrial deflections was a characteristic finding in AMA-M2-positive patients with supraventricular arrhythmias.

Conclusion AMA-M2 enhances the risk of supraventricular arrhythmias, indicating the possible involvement of the atrial myocardium and the formation of an arrhythmogenic substrate. The results highlight the need for clinical attention to supraventricular arrhythmias in AMA-M2-positive patients.
\end{abstract}

Key words: anti-mitochondrial antibody, cardiomyopathy, primary biliary cholangitis, supraventricular arrhythmia

(Intern Med 56: 1771-1779, 2017)

(DOI: 10.2169/internalmedicine.56.8183)

\section{Introduction}

Anti-mitochondrial antibodies (AMAs) are autoantibodies against several mitochondrial antigens that can be detected in the sera from patients with primary biliary cholangitis (PBC) by immunoblotting and enzyme-linked immunosorbent assays (1). To date, nine mitochondrial antigens (M1M9) have been reported to stimulate the production of the corresponding antibodies, AMA-M1 to AMA-M9 (2).

${ }^{1}$ Division of Cardiovascular Medicine, Department of Internal Medicine, Kobe University Graduate School of Medicine, Japan, ${ }^{2}$ Section of Arrhythmia, Division of Cardiovascular Medicine, Department of Internal Medicine, Kobe University Graduate School of Medicine, Japan and ${ }^{3}$ Division of Gastroenterology, Department of Internal Medicine, Kobe University Graduate School of Medicine, Japan Received for publication August 29, 2016; Accepted for publication November 28, 2016 Correspondence to Dr. Koji Fukuzawa, kfuku@med.kobe-u.ac.jp 
Among these antigens, AMA-M2 reacts with the pyruvate dehydrogenase E2 complex. This target antigen is located in the inner mitochondrial matrix and catalyzes the oxidative decarboxylation of keto acid substrates $(3,4)$. AMA-M2 is detected in $90-95 \%$ of patients with PBC (4). Skeletal muscular involvement manifesting as progressive muscular weakness with elevated levels of muscle-associated enzymes and respiratory failure has been reported in patients with AMA-M2 (5-7). Although the mechanism underlying the development of AMA-M2-associated myopathy has yet to be clarified, it is considered likely that mitochondrial dysfunction and autoimmunity, which are maintained by the predominant autoreactive $\mathrm{T}$ cells, are associated with the skeletal muscular damage (8). Furthermore, concomitant cardiac involvement has been reported in patients with AMA-M2associated myopathy, as revealed by ventricular dysfunction and/or various arrhythmias $(5,6)$. Among patients with such arrhythmias, the prevalence of supraventricular arrhythmias has been reported to be high (5), which might suggest a preferential atrial myocardial involvement. However, the prevalence of supraventricular arrhythmias in unselected AMA-M2-positive patients has yet to be systematically investigated in the clinical setting. Accordingly, little is known regarding the clinical impact of AMA-M2 on supraventricular arrhythmias. The objective of this study was to clarify the prevalence of supraventricular arrhythmias in AMA-M2positive patients, regardless of skeletal muscular involvement. The impact of AMA-M2 on supraventricular arrhythmias was then investigated to estimate the atrial myocardial involvement and the formation of arrhythmogenic substrates.

\section{Materials and Methods}

\section{Study population}

We retrospectively analyzed 384 consecutive patients who underwent AMA-M2 testing for the further examination of elevated hepatobiliary enzyme levels between August 2012 and July 2014.

Elevated hepatobiliary enzyme levels were defined by any of the following conditions: aspartate aminotransferase $($ AST) $>32 \mathrm{IU} / \mathrm{L}$, alanine aminotransferase $($ ALT) $>37$ IU/L, alkaline phosphatase (ALP) $>100$ IU/L, or $\gamma$-glutamyltranspeptidase $(\gamma$-GTP) $>51$ IU/L. In all cases, the clinical history was acquired and a laboratory analysis was performed. Congestive heart failure was defined by the presence of symptoms of heart failure classified as New York Heart Association class $\geq$ II or a left ventricular ejection fraction of $<40 \%$. Hypertension was defined as a systolic blood pressure of $\geq$ $140 \mathrm{mmHg}$, a diastolic pressure of $\geq 90 \mathrm{mmHg}$, or the use of antihypertensive agents. Diabetes mellitus was defined as a fasting glucose level of $\geq 126 \mathrm{mg} / \mathrm{dL}$, a random non-fasting glucose level of $\geq 200 \mathrm{mg} / \mathrm{dL}$, a hemoglobin A1c level of $\geq$ $6.5 \%$, or the use of oral hypoglycemic agents or insulin. The $\mathrm{CHADS}_{2}$ score was generated by assigning one point each for the following factors: a history of congestive heart fail- ure, hypertension, age $\geq 75$ years, and diabetes mellitus, and by assigning two points for a history of stroke or transient ischemic attack (9).

The present study was performed according to standard clinical practice and complied with the Declaration of Helsinki. All of the study participants provided their informed consent. The research protocol was approved by the Human Ethical Committee at Kobe University Hospital (No. 1830).

\section{Laboratory analysis}

The following clinical laboratory parameters were evaluated: white blood cell count, hemoglobin, platelet count; liver function parameters (including total bilirubin, AST, ALT, ALP, and $\gamma$-GTP); creatinine kinase (CK); albumin; electrolytes (including sodium, potassium, and chloride); renal function parameters (including blood urea nitrogen, creatinine, and the estimated glomerular filtration rate [eGFR]); C-reactive protein, and AMA-M2. The eGFR was obtained from the equation defined by the Japanese Society of Nephrology: eGFR $=194 \times$ serum creatinine level $(\mathrm{mg} / \mathrm{dL})^{-1.094} \times$ age (years) ${ }^{-0.287}$ (if female $\left.\times 0.739\right) \mathrm{mL} / \mathrm{min} / 1.73 \mathrm{~m}^{2}(10)$.

The AMA-M2 titer was evaluated using a commercially available chemiluminescent enzyme immuno-assay (Medical \& Biological Laboratories, Nagoya, Japan). AMA-M2 positivity was defined as an AMA-M2 titer of $\geq 7.0 \mathrm{IU} / \mathrm{mL}$.

\section{Definition of arrhythmias}

Arrhythmias were detected based on a 12-lead electrocardiogram (ECG), 24-hour ambulatory monitoring, and/or a physician-assigned diagnosis that was found in the medical records within three years before and two years after AMAM2 testing. Furthermore, asymptomatic arrhythmias documented on ECG monitoring during hospitalization and in implanted devices were considered to be arrhythmias. In this situation, arrhythmias lasting for $>30$ seconds were included.

Supraventricular arrhythmias were defined as supraventricular tachyarrhythmias involving atrial fibrillation (AF), atrial flutter (AFL), and atrial tachycardia (AT), and supraventricular bradyarrhythmias involving sick sinus syndrome (SSS) and atrial standstill. AF was defined as irregular R-R intervals with typical fibrillation in the baseline without distinct P-waves. AFL was defined as the presence of characteristic regular flutter waves with a cycle length of $\leq 250 \mathrm{~ms}$ without an isoelectric baseline between the atrial deflections in at least a single lead. AT was defined as a regular ectopic P-wave with a cycle length of $\leq 400 \mathrm{~ms}$ and distinct P-waves that differed from normal sinus rhythm. SSS was defined as sinus bradycardia $<50 \mathrm{bpm}$, sinus arrest of $>3.0$ seconds, and/or sinoatrial exit block. Atrial standstill was defined as the continuous disappearance of any atrial deflections in the baseline with an escape rhythm, representing the electrical or mechanical silence of the atrial excitation. Ventricular arrhythmias were defined as sustained ventricular tachycardia lasting for $>30$ seconds or the detection of ventricular fibrillation on a device recording. 
Table 1. Comparison of Baseline Characteristics.

\begin{tabular}{|c|c|c|c|}
\hline Variable & $\begin{array}{l}\text { AMA-M2 positive group } \\
\qquad \mathrm{n}=77\end{array}$ & $\begin{array}{l}\text { AMA-M2 negative group } \\
\qquad \mathrm{n}=307\end{array}$ & $\mathrm{p}$ value \\
\hline Age, years & $60(51-69)$ & $60(47-70)$ & 0.512 \\
\hline Male gender, n (\%) & $4(5)$ & $112(36)$ & $<0.001$ \\
\hline Body mass index, $\mathrm{kg} / \mathrm{m}^{2}$ & $21.9(18.8-25.4)$ & $21.9(19.3-24.6)$ & 0.937 \\
\hline Congestive heart failure, $\mathrm{n}(\%)$ & $7(9)$ & $13(4)$ & 0.082 \\
\hline Hypertension, n (\%) & $24(31)$ & $85(28)$ & 0.545 \\
\hline Age $\geq 75, \mathrm{n}(\%)$ & $8(10)$ & $41(13)$ & 0.314 \\
\hline Diabetes mellitus, n (\%) & $11(14)$ & $74(24)$ & 0.064 \\
\hline Stroke/transient ischemic attack, $\mathrm{n}(\%)$ & $1(1)$ & $7(2)$ & 0.500 \\
\hline $\mathrm{CHADS}_{2}$ score & $0(0-1)$ & $1(0-1)$ & 0.404 \\
\hline
\end{tabular}

Nonparametric continuous variables are expressed as median values (lower quartile value-upper quartile value).

AMA-M2: anti-mitochondrial antibody M2

\section{Baseline 12-lead ECG}

We hypothesized that if significant latent atrial muscular damage due to AMA-M2 was present, it could be reflected in the baseline 12-lead ECG findings as a low P-wave amplitude. To evaluate this type of latent electrical remodeling in either the atrium or ventricle, we retrospectively analyzed the subgroup of patients with 12-lead ECG data by investigating the following parameters of the baseline 12-lead ECG. In addition to the heart rate, the PR interval, P-wave amplitude, P-wave duration, Morris index, QRS duration, QRS axis, QT interval, corrected QT interval, sum of the amplitude of the S-wave in lead V1 and the R-wave in lead V5 (SV1+RV5), and the prevalence of P-dextrocardiale and $\mathrm{P}$-sinistrocardiale were measured. We also analyzed the presence of complete right or left bundle branch block.

The P-wave amplitude, $\mathrm{P}$-wave duration, and Morris index were measured using lead V1. The Morris index was derived by multiplying the depth of the terminal P-wave deflection $(\mathrm{mm})$ by its duration (s). The P-dextrocardiale was defined as a P-wave-positive component of $\geq 0.25 \mathrm{mV}$ in leads II, III, and aVF, or a P-wave-positive component of $\geq 0.2 \mathrm{mV}$ in lead V1 or V2, or a P-wave duration of $\leq 100 \mathrm{~ms}$ in lead V1, reflecting the overloading of the right atrium. The Psinistrocardiale was defined as a Morris index of $\geq 0.04$ or a P-wave duration of $\geq 120 \mathrm{~ms}$ in lead II, reflecting the overloading of the left atrium.

\section{Transthoracic echocardiography}

Based on the hypothesis that latent mechanical remodeling of either the atrium or ventricle could be detected as morphological changes, we retrospectively analyzed the subgroup of patients for whom echocardiographic data was available. All of the transthoracic echocardiographic studies were performed using commercially available echocardiographic systems (Aplio Artida, Toshiba Medical Systems, Tochigi, Japan; Vivid E9, GE Vingmed, Horten, Norway; iE 33, Philips Healthcare, Andover, USA). Using the standard parasternal view, we measured the dimensions of the left ventricle and the left atrium according to the American Soci- ety of Echocardiography guidelines (11). Each value was indexed by dividing it by the body surface area. The early diastolic (E) and atrial filling (A) wave velocities of the transmitral inflow, as well as E-wave deceleration time and E/A, were measured using a pulsed-wave Doppler recording in the standard apical three- or four-chamber view.

\section{Statistical analysis}

Nonparametric continuous variables were expressed as median values with interquartile ranges. Categorical variables were expressed as a percentage. Intergroup differences were evaluated using the Mann-Whitney U test for nonparametric continuous variables and a chi-squared test for categorical variables. The univariate relationships between the supraventricular arrhythmias and variables were evaluated by a logistic regression analysis. A multivariate logistic regression analysis was performed using the maximum likelihood method to identify the independent risk factors for supraventricular arrhythmias. The association was shown as an odds ratio with $95 \%$ confidence intervals and $p$ values. All of the statistical analyses were performed using the SPSS software program (version 20.0; IBM, Chicago, USA). p values of $<0.05$ were considered to indicate statistical significance in all of the analyses.

\section{Results}

In total, 384 patients (116 men; median age, 60 [48-69] years) were enrolled in this study. Seventy-seven of the patients were positive for AMA-M2. Comparisons of the baseline characteristics of the AMA-M2-positive and AMA-M2negative patients are shown in Table 1. The proportion of male patients was significantly lower among the AMA-M2positive patients. The $\mathrm{CHADS}_{2}$ scores of the two groups did not differ to a statistically significant extent.

Among the laboratory parameters, the white blood cell count, hemoglobin level, and eGFR were significantly lower, and the level of alanine aminotransferase was significantly higher in the AMA-M2-positive patients. No significant differences were documented for the other laboratory parame- 
Table 2. Comparison of Laboratory Parameters.

\begin{tabular}{lccc}
\hline \multicolumn{1}{c}{ Variable } & AMA-M2 positive group & AMA-M2 negative group & $\mathrm{p}$ value \\
& $\mathrm{n}=77$ & $\mathrm{n}=307$ & \\
\hline White blood cell, $\times 10^{2} / \mu \mathrm{L}$ & $56(46-65)$ & $12.8(11.3-14.0)$ & 0.042 \\
Hemoglobin, g/dL & $12.5(11.2-13.2)$ & $21.6(16.0-26.9)$ & 1.000 \\
Platelet, $\times 10^{4} / \mu \mathrm{L}$ & $21.3(17.1-26.8)$ & $0.7(0.5-1.0)$ & 0.441 \\
Total bilirubin, mg/dL & $0.7(0.6-1.0)$ & $37(25-63)$ & 0.984 \\
Aspartate aminotransferase, IU/L & $37(25-58)$ & $38(23-71)$ & 0.011 \\
Alanine aminotransferase, IU/L & $27(19-53)$ & $307(229-466)$ & 0.920 \\
Alkaline phosphatase, IU/L & $306(214-464)$ & $57(29-148)$ & 0.503 \\
$\gamma$-glutamyltranspeptidase, IU/L & $72(28-152)$ & $81(46-133)$ & 0.782 \\
Creatinine kinase, IU/L & $72(47-140)$ & $4.0(3.4-4.4)$ & 0.714 \\
Albumin, g/dL & $4.1(3.4-4.3)$ & $140(138-141)$ & 0.254 \\
Sodium, mEq/L & $140(139-142)$ & $4.1(3.8-4.4)$ & 0.943 \\
Potasssium, mEq/L & $4.1(3.8-4.3)$ & $105(103-107)$ & 0.227 \\
Chloride, $\mathrm{mEq} / \mathrm{L}$ & $105(104-107)$ & $14.0(11.0-17.0)$ & 0.881 \\
Blood urea nitrogen, mg/dL & $14.0(11.1-17.4)$ & $0.68(0.56-0.84)$ & 0.421 \\
Creatinine, mg/dL & $0.62(0.58-0.78)$ & $77.7(63.5-93.5)$ & 0.043 \\
eGFR, mL/min/1.73 m ${ }^{2}$ & $74.0(57.1-85.2)$ & $0.14(0.56-0.84)$ & 0.825 \\
C-reactive protein, mg/dL & $0.15(0.58-0.78)$ & $1.4(1.4-4.9)$ & $<0.001$ \\
AMA-M2 titer, IU/mL & $106.0(20.3-195.0)$ & & \\
\hline
\end{tabular}

Nonparametric continuous variables are expressed as median values (lower quartile value-upper quartile value). AMA-M2: anti-mitochondrial antibody M2, eGFR: estimated glomerular filtration rate

ters, including CK (Table 2).

The baseline 12-lead ECG data were available for 45 AMA-M2-positive (58\%) and 202 AMA-M2-negative (66\%) patients (Table 3). The 24-hour ambulatory monitoring data was available for four AMA-M2-positive and 17 AMA-M2negative patients. The ECG monitoring data recorded during hospitalization was available for 21 AMA-M2-positive patients and 115 AMA-M2-negative patients. The ECG records from implanted devices were available for four AMA-M2positive patients and three AMA-M2-negative patients.

Supraventricular arrhythmias were detected in $14 \%$ of the AMA-M2-positive patients, which was significantly higher than that in the AMA-M2-negative patients (Table 3). Among these patients, symptomatic supraventricular arrhythmias were documented in seven AMA-M2-positive and 10 AMA-M2-negative patients. Conversely, the frequency of ventricular arrhythmias was much lower than that of supraventricular arrhythmias and did not differ between the two groups. Among the 11 AMA-M2-positive patients with supraventricular arrhythmias, eight patients showed AF. AFL and AT were each detected in two and four patients, respectively. SSS was detected in three patients. Atrial standstill was detected in four of the 11 patients. It was notable that four patients had multiple supraventricular arrhythmias, including both tachyarrhythmias and bradyarrhythmias.

A comparison of the baseline 12-lead ECG findings revealed that the QRS duration and QRS axis were significantly lower in AMA-M2-positive patients (Table 3).

The baseline echocardiography was available for 31 AMA-M2-positive (40\%) and 124 AMA-M2-negative (40\%) patients. Although the left ventricular dimension indices and ejection fraction of the AMA-M2-positive and AMA-M2- negative patients were similar, the left atrial end-systolic dimension index was significantly larger in the AMA-M2positive patients (Table 4), suggesting the existence of possible left atrial remodeling.

Univariate analyses were performed to assess the relationship between each variable and the supraventricular arrhythmias (Table 5). The variables with $p$ values of $<0.2$ in the comparison of patients with and without supraventricular arrhythmias (data not shown) were included in this analysis. The univariate analysis showed that aging, congestive heart failure, a high $\mathrm{CHADS}_{2}$ score, and AMA-M2 positivity were significantly correlated with supraventricular arrhythmias.

To ascertain the potential independent risk factors for supraventricular arrhythmias in the present patient cohort, we next performed a multivariate logistic regression analysis. Variables with $\mathrm{p}$ values $<0.05$ in the univariate model were included in the final multivariate model. The $\mathrm{CHADS}_{2}$ score was excluded to avoid multicollinearity. As previous reports demonstrated, supraventricular arrhythmias were observed in the limited subset of patients with AMA-M2-associated myopathy, CK was also included in this model. This analysis showed that the presence of AMA-M2, congestive heart failure and creatinine kinase were independent risk factors for supraventricular arrhythmias (Table 6).

The AMA-M2 titer of the AMA-M2-positive patients, did not differ to a statistically significant extent, regardless of the presence or absence of supraventricular arrhythmias (4.9 [1.4-20.9] IU/mL vs. 3.0 [1.4-4.9] IU/mL, p=0.777).

Few reports have described the detailed characteristics of supraventricular arrhythmias in AMA-M2-positive patients. Thus, we closely analyzed the supraventricular arrhythmias in AMA-M2-positive patients. Our clinical observations re- 
Table 3. Comparison of the Electrocardiographic Findings.

\begin{tabular}{lccc}
\hline \multicolumn{1}{c}{ Variable } & $\begin{array}{c}\text { AMA-M2 positive patients } \\
\mathrm{n}=77\end{array}$ & $\begin{array}{c}\text { AMA-M2 negative patients } \\
\mathrm{n}=307\end{array}$ & $\mathrm{p}$ value \\
\hline Supraventricular arrhythmias, $\mathrm{n}(\%)$ & $11(14)$ & $17(6)$ & 0.008 \\
Atrial fibrillation, $\mathrm{n}(\%)$ & $8(10)$ & $13(4)$ & \\
Atrial flutter, $\mathrm{n}(\%)$ & $2(3)$ & $3(1)$ & \\
Atrial tachycardia, $\mathrm{n}(\%)$ & $4(5)$ & $0(0)$ & \\
Sick sinus syndorome, $\mathrm{n}(\%)$ & $3(4)$ & $2(1)$ & \\
Atrial standstill, $\mathrm{n}(\%)$ & $4(5)$ & $1(0)$ & 0.181 \\
Ventricular arrhythmias, $\mathrm{n}(\%)$ & $2(3)$ & $2(1)$ & \\
& & & $\mathrm{n}=202$ \\
12-lead electrocardiogram & $\mathrm{n}=45$ & $13(6)$ & 0.054 \\
Supraventricular arrhythmias, $\mathrm{n}(\%)$ & $7(16)$ & $73(65-82)$ & 0.288 \\
Heart rate, beats/min & $70(65-79)$ & $158(142-174)$ & 0.668 \\
PR interval, ms & $156(142-176)$ & $0.08(0.06-0.10)$ & 0.140 \\
P-wave amplitude, mV & $0.09(0.06-0.12)$ & $80(65-90)$ & 0.578 \\
P-wave duration, ms & $80(70-90)$ & $0.017(0.007-0.030)$ & 0.546 \\
Morris index, mm·sec & $0.018(0.009-0.030)$ & $90(83-100)$ & 0.033 \\
QRS duration, ms & $86(80-92)$ & $46(22-67)$ & 0.027 \\
QRS axis, degree & $32(-9-66)$ & $389(366-410)$ & 0.235 \\
QT interval, ms & $398(374-415)$ & $416(398-430)$ & 0.311 \\
QTc, ms & $422(401-432)$ & $2.39(1.95-2.97)$ & 0.930 \\
SV1+RV5, mV & $2.50(1.89-2.90)$ & $1(0)$ & 0.817 \\
P-dextrocardiale, $\mathrm{n}(\%)$ & $0(0)$ & $12(3)$ & 0.549 \\
P-sinistrocardiale, $\mathrm{n}(\%)$ & $1(2)$ & $1(0)$ & 0.547 \\
Complete right bundle branch block, $\mathrm{n}(\%)$ & $2(4)$ & 0.827 \\
Complete left bundle branch block, $\mathrm{n}(\%)$ & $0(0)$ & & \\
\hline
\end{tabular}

Nonparametric continuous variables are expressed as median values (lower quartile value-upper quartile value).

AMA-M2: anti-mitochondrial antibody M2, QTc: corrected QT interval, SV1+RV5: the sum of the amplitude of S-wave in V1 lead and R- wave in V5 lead

Table 4. Comparison of the Echocardiographic Findings.

\begin{tabular}{lccc}
\hline \multicolumn{1}{c}{ Variable } & AMA-M2 positive patients & AMA-M2 negative patients & $\mathrm{p}$ value \\
& $\mathrm{n}=77$ & $\mathrm{n}=307$ & \\
\hline Echocardiography & $\mathrm{n}=31$ & $12(10)$ & 0.013 \\
Supraventricular arrhythmias, $\mathrm{n}(\%)$ & $9(29)$ & $28.6(26.4-31.7)$ & 0.974 \\
Left ventricular end-diastolic dimension index, $\mathrm{mm} / \mathrm{m}^{2}$ & $29.3(25.2-31.9)$ & $18.1(15.9-20.8)$ & 0.753 \\
Left ventricular end-systolic dimension index, $\mathrm{mm} / \mathrm{m}^{2}$ & $17.2(15.8-21.8)$ & $36.5(32.4-41.0)$ & 0.708 \\
Fractional shortening, \% & $38.6(31.9-41.4)$ & $22.6(19.9-25.5)$ & 0.001 \\
Left atrial end-systolic dimension index, $\mathrm{mm} / \mathrm{m}^{2}$ & $26.2(22.1-28.7)$ & $9.8(8.5-11.0)$ & 0.727 \\
Interventricular septal wall thickness, mm & $9.4(8.1-11.5)$ & $9.8(8.1-11.0)$ & 0.771 \\
Left ventricular inferolateral wall thickness, $\mathrm{mm}$ & $9.7(8.5-10.8)$ & $65.0(60.0-67.8)$ & 0.176 \\
Left ventricular ejection fraction, \% & $66.2(60.1-71.3)$ & & \\
Transmitral flow & & $66.9(53.8-80.6)$ & 0.185 \\
Early diastolic filling (E) wave velocity, $\mathrm{cm} / \mathrm{s}$ & $66.8(60.6-93.0)$ & $67.8(57.3-84.6)$ & 0.024 \\
Atrial filling (A) wave velocity, cm/s & $77.4(66.6-108.0)$ & $0.90(0.70-1.20)$ & 0.937 \\
E/A & $0.90(0.75-1.10)$ & $208(174-262)$ & 0.375 \\
E-wave deceleration time, $\mathrm{ms}$ & $227(192-276)$ & $0(0)$ & 0.202 \\
Severe mitral regurgitation, $\mathrm{n}(\%)$ & $1(3)$ & & \\
\hline
\end{tabular}

Nonparametric continuous variables are expressed as median values (lower quartile value-upper quartile value).

AMA-M2: anti-mitochondrial antibody M2

vealed the following characteristics: 1) multiple supraventricular arrhythmias in a single patient, 2) the coincidence of tachyarrhythmias and bradyarrhythmias, 3) extremely low atrial deflections, and 4) frequent AF. In particular, the aver- age value of the atrial deflections in the AMA-M2-positive AF patients was extremely low $(0.03 \pm 0.01 \mathrm{mV})$. Figure shows a representative case of an AMA-M2-positive patient (a 67-year-old woman) with multiple supraventricular ar- 
Table 5. Univariate Relationship between Variables and the Supraventricular Arrhythmias.

\begin{tabular}{lccc}
\hline \multicolumn{1}{c}{ Variable } & Odds ratio & $95 \%$ CI & p value \\
\hline Age (per decade increase) & 1.45 & $1.01-1.07$ & 0.015 \\
Body mass index & 0.92 & $0.82-1.02$ & 0.108 \\
Congestive heart failure & 4.94 & $1.65-14.80$ & 0.004 \\
Hypertension & 2.00 & $0.91-4.39$ & 0.083 \\
CHADS 2 score & 1.51 & $1.06-2.17$ & 0.025 \\
Hemoglobin & 0.83 & $0.69-1.00$ & 0.051 \\
Platelet & 0.99 & $0.95-1.04$ & 0.763 \\
Total bilirubin & 0.49 & $0.18-1.40$ & 0.184 \\
Alanine aminotransferase (per 10 IU/L elevation) & 0.92 & $0.82-1.03$ & 0.143 \\
Alkaline phosphatase (per 10 IU/L elevation) & 0.99 & $0.98-1.01$ & 0.351 \\
Creatinine kinase (per 50 IU/L elevation) & 1.03 & $1.00-1.05$ & 0.054 \\
Chloride (per 10 mEq/L elevation) & 0.47 & $0.18-1.22$ & 0.121 \\
Blood urea nitrogen (per 10 mg/dL elevation) & 1.30 & $0.98-1.71$ & 0.069 \\
AMA-M2 & 2.84 & $1.27-6.35$ & 0.011 \\
\hline
\end{tabular}

AMA-M2: anti-mitochondrial antibody M2, CI: confidence interval

Table 6. Independent Predictors of Supraventricular Arrhythmias.

\begin{tabular}{lccc}
\hline \multicolumn{1}{c}{ Variable } & Odds ratio & $95 \%$ CI & p value \\
\hline Age (per decade increase) & 1.42 & $1.00-2.04$ & 0.053 \\
Congestive heart failure & 4.87 & $1.35-17.65$ & 0.016 \\
Creatinine kinase (per 50 IU/L elevation) & 1.03 & $1.00-1.06$ & 0.028 \\
AMA-M2 & 3.52 & $1.34-9.24$ & 0.011 \\
\hline
\end{tabular}

AMA-M2: anti-mitochondrial antibody M2, CI: confidence interval

rhythmias, in whom AF, AT, and atrial standstill with dilated atria were present. Notably, the voltage of the atrial deflections in these multiple supraventricular arrhythmias was extremely low, which suggested an extensive atrial arrhythmogenic substrate due to substantial and progressive AMA-M2associated damage to the atrial myocardium.

\section{Discussion}

Here, for the first time, we clarify the prevalence of supraventricular arrhythmias in unselected AMA-M2-positive patients. The prevalence of supraventricular arrhythmias in AMA-M2-positive patients was $14 \%$, which was significantly higher than that in AMA-M2-negative patients. The multivariate analysis revealed that the presence of AMA-M2 was an independent risk factor for supraventricular arrhythmias in the present patient cohort. The supraventricular arrhythmias that were associated with AMA-M2 were also characterized.

\section{The association between AMA-M2 and supraven- tricular arrhythmias}

To date, limited data have been available on the independent association between AMA-M2 and supraventricular arrhythmias. Maeda et al. reported 5 (21\%) patients with supraventricular arrhythmias among 24 patients with AMAM2-associated myopathy (5). In contrast to their cohort, we included all of the AMA-M2-positive patients, irrespective of their history of myopathy or CK values, in order to determine the prevalence of supraventricular arrhythmias in unselected AMA-M2-positive patients. Supraventricular arrhythmias were found in $14 \%$ of the AMA-M2-positive patients. As shown in Table 2, the CK level did not differ to between the AMA-M2-positive and AMA-M2-negative patients, suggesting that our patient cohort did not include a substantial number of patients with clinically significant AMA-M2associated myopathy. Furthermore, the multivariate analyses revealed that AMA-M2 was associated with supraventricular arrhythmias, even when the CK level was included in the model (Table 6). Considering these results, AMA-M2 appears to be independently associated with supraventricular arrhythmias, irrespective of skeletal muscular involvement.

The AMA-M2 titer of the AMA-M2-positive patients did not differ to a statistically significant extent, regardless of the absence or presence of supraventricular arrhythmias. In contrast, a previous study reported that arrhythmias tended to be more frequent in patients with a high AMA-M2 titer (5). Thus, further observation is required to clarify the association between the AMA-M2 titer and the progression of cardiac involvement, including the frequency of supraventricular arrhythmias.

\section{The preferential involvement of the atrium}

In the present study, the prevalence of supraventricular arrhythmias was higher than that of ventricular arrhythmias; this result was compatible with a previous report (5). This 
A

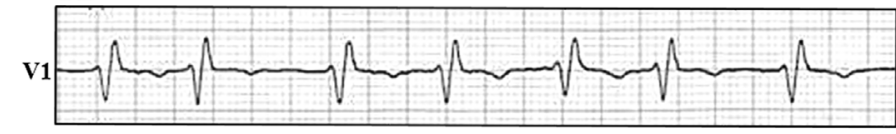

B

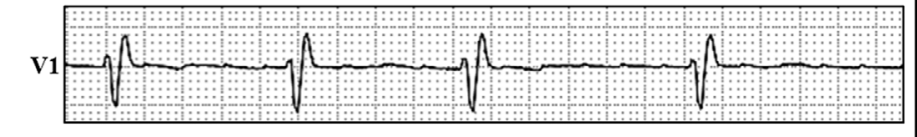

v1
D

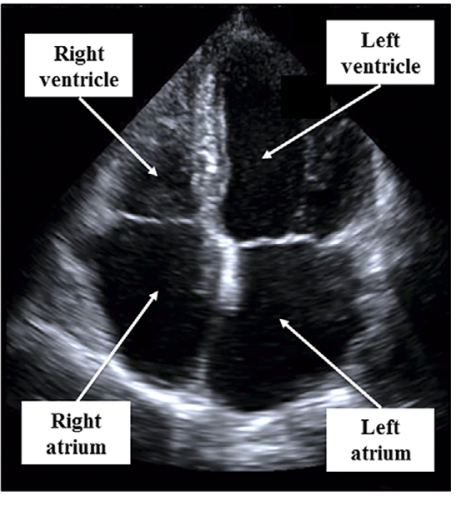

Figure. A representative case of multiple supraventricular arrhythmias in an anti-mitochondrial M2 antibody-positive patient. (A) A V1 lead electrocardiogram showing atrial fibrillation with low amplitude fibrillation waves. (B) Atrial tachycardia is confirmed in the V1 lead. Note the low amplitude of the regular ectopic P-waves. (C) Atrial standstill with an escape rhythm demonstrated in the V1 lead. (D) The apical four-chamber view of the echocardiographic examination in systole showing dilation of both atria (left atrial diameter: $53 \times 61 \mathrm{~mm}$, right atrial diameter: $49 \times 60 \mathrm{~mm}$ ).

suggests that substantial degeneration occurred in the atrial myocardium, which might work as a substrate for supraventricular arrhythmias. To the best of our knowledge, however, few reports have demonstrated atrial myocardial involvement in a convincing fashion. We consider that a high prevalence of supraventricular arrhythmias might, in part, be explained by the difference in the myocardial wall between the atria and ventricles. As the atrial wall is considerably thinner than the ventricular wall, it might be susceptible to a smaller degree of damage, manifesting as the preferential involvement of the atrium. The left atrial enlargement that was observed in AMA-M2-positive patients (Table 4) may support this hypothesis.

\section{A possible mechanism of AMA-M2-associated myo- cardial involvement and supraventricular arrhyth- mias}

Among the nine AMAs, AMA-M7 has been detected in patients with acute and chronic cardiomyopathies $(12,13)$. AMA-M2 was also found in some cardiomyopathy patients $(6,7)$. Notably, Varga et al. described the presence of skeletal mitochondrial damage without significant inflammation in PBC patients (14), suggesting a direct disturbance of the muscular energy metabolism by the AMAs. Conversely, several reports have demonstrated inflammation due to cellular immunological mechanisms (15). In particular, Matsumoto et al. revealed the interstitial infiltration of CD3positive $T$ cells in the ventricular myocardium of a patient with AMA-M2-associated cardiomyopathy (6), and Shimizu et al. reported the predominant infiltration of CD4-positive T cells in the skeletal muscles of a patient with AMA-M2associated myopathy (8). Although the mechanism of AMAM2-associated myopathy has yet to be clarified, these observations suggest that mitochondrial dysfunction due to humoral autoimmunity and cellular autoimmunity maintained by autoreactive $\mathrm{T}$ cells may be involved in the mechanism of AMA-M2-associated myocardial involvement and the resultant supraventricular arrhythmias. In addition, individual variations in the immunological system and certain viral infections may also play important roles in producing pathogenic autoantibodies, which might trigger chronic autoimmunity against multiple organs (16).

Finally, Keresztes et al. reported that autonomic nerve dysfunction is a frequent complication in PBC patients (17). Specifically, their findings from an analysis of 24-hour heart rate variability indicated a synchronic impairment of the parasympathetic and sympathetic systems. As the autonomic nervous system is closely associated with the occurrence of AF and SSS (18), autonomic nerve dysfunction might influence the triggering of supraventricular arrhythmias in AMAM2-positive patients.

\section{Clinical implications}

The results of this study demonstrated that the presence of AMA-M2 is an independent risk factor for supraventricular arrhythmias, including AF, AFL, AT, SSS, and atrial standstill. Although we were unable to confirm the latent electrical remodeling of the atria by our subgroup analysis of the 12-lead ECG data of the AMA-M2-positive patients (Table 3), periodical ECGs are recommended to avoid missing any asymptomatic supraventricular arrhythmias. Considering the fact that a number of AMA-M2-positive patients associated with PBC are currently managed by hepatologists, referral to cardiology should be considered in patients with arrhythmias. In turn, cardiologists need to suspect AMA-M2-associated myopathy and PBC, when middle-aged women with elevated hepatobiliary enzymes present with characteristic supraventricular arrhythmias, as shown in Figure.

Based on the supposition that AMA-M2 related atrial in- 
volvement would likely be progressive, our finding suggested a potential need for anticoagulation therapy or pacemaker implantation during the clinical course of patients who are positive for AMA-M2. To date, however, no guidelines that have focused on these supraventricular arrhythmias exist for the management of AMA-M2-positive patients. We therefore highlight the need for physicians to pay attention to supraventricular arrhythmias in AMA-M2-positive patients. Considering the likelihood that autoimmunity might be involved in the development of these supraventricular arrhythmias, immunosuppressive therapy, including glucocorticoids (6), might represent a better therapeutic option for suppressing these arrhythmias than anti-arrhythmic agents. If used, immunosuppressive therapy should be initiated as early as possible to limit disease progression.

The present results also highlighted a need for physicians to update their knowledge of AMAs, as well as to keep abreast of the current nomenclature and guidelines for the disease entity that is referred to as "primary biliary cirrhosis". The term, "primary biliary cirrhosis," is being replaced by "primary biliary cholangitis" because the conventional term does not accurately reflect the natural history of disease in the vast majority of patients (19). In the latest guidelines, histological findings are not necessarily indispensable in the diagnosis of PBC. The presence of AMAs accompanied by clinical features and a course of classical and cholestatic PBC are required, including increased levels of serum ALP and $\gamma$-GTP (20).

\section{Study limitations}

Our study is associated with several potential limitations. First, a selection bias could not be avoided in our patient cohort because the present observational study was conducted retrospectively. In particular, the 12-lead ECG and echocardiographic data were retrospectively available for only $64 \%$ and $40 \%$ of the patients, respectively. The subgroup results in Tables 3 and 4 should therefore be confirmed in future studies. Second, because the present study was conducted in a single center, the patient population was relatively small. Thus, the present results may not be representative of AMA-M2-positive patients in the general population. Our results should therefore be verified using a prospective and multi-center study design. Third, supraventricular arrhythmias are frequently asymptomatic. Thus, we cannot deny the possibility of the underestimation of their prevalence. Finally, although our close observation of supraventricular arrhythmias and the echocardiographic subanalysis suggested extensive atrial myocardial damage, we were unable to perform a biopsy of the atrial thinner musculature in most cases and it was difficult to directly detect inflammation or fibrosis of the atrium. Further studies based on intraoperatively obtained specimens, autopsy results, electroanatomic mapping, ${ }^{18} \mathrm{~F}$ isotope-labeled positron emission tomography, metaiodobenzylguanidine myocardial scintigraphy, and delayed enhancement magnetic resonance imaging would likely have the potential to elucidate the mechanism of supraventricular arrhythmias in patients with AMA-M2-associated cardiomyopathy.

\section{Conclusion}

The present study revealed that the prevalence of supraventricular arrhythmias represented one seventh of the patients with positive AMA-M2 test results. Furthermore, AMA-M2 conferred a substantial risk of supraventricular arrhythmias, suggesting that atrial myocardial involvement and the formation of an arrhythmogenic substrate. Physicians should maintain awareness of supraventricular arrhythmias in AMA-M2-positive patients.

\section{Author's disclosure of potential Conflicts of Interest (COI).}

Koji Fukuzawa: Research funding, Medtronic Japan and St. Jude Medical Japan. Kunihiko Kiuchi: Research funding, Medtronic Japan and St. Jude Medical Japan. Ken-ichi Hirata: Research funding, Medtronic Japan and St. Jude Medical Japan.

\section{References}

1. Frostell A, Mendel-Hartvig I, Nelson BD, et al. Mitochondrial autoantigens in primary biliary cirrhosis. Association of diseasespecific determinants with a subunit of complex I (NADHubiquinone reductase) of the inner mitochondrial membrane. Scand J Immunol 28: 645-652, 1988.

2. Berg PA, Klein R. Mitochondrial antigens and autoantibodies: from anti-M1 to anti-M9. Klin Wochenschr 64: 897-909, 1986.

3. Bassendine MF, Jones DE, Yeaman SJ. Biochemistry and autoimmune response to the 2-oxoacid dehydrogenase complexes in primary biliary cirrhosis. Semin Liver Dis 17: 49-60, 1997.

4. Kaplan MM, Gershwin ME. Primary biliary cirrhosis. N Engl J Med 353: 1261-1273, 2005.

5. Maeda MH, Tsuji S, Shimizu J. Inflammatory myopathies associated with anti-mitochondrial antibodies. Brain 135: 1767-1777, 2012.

6. Matsumoto K, Tanaka H, Yamana S, et al. Successful steroid therapy for heart failure due to myocarditis associated with primary biliary cirrhosis. Can J Cardiol 28: 515 e3-e6, 2012.

7. Uenaka T, Kowa H, Sekiguchi K, et al. Myositis with antimitochondrial antibodies diagnosed by rectus abdominis muscle biopsy. Muscle Nerve 47: 766-768, 2013.

8. Shimizu J. Clinical and histopathological feature of myositis associated with anti-mitochondrial antibodies. Rinsho Shinkeigaku. Clin Neurol 53: 1114-1116, 2013 (in Japanese, Abstract in English).

9. Gage BF, Waterman AD, Shannon W, et al. Validation of clinical classification schemes for predicting stroke: results from the National Registry of Atrial Fibrillation. JAMA 285: 2864-2870, 2001.

10. Japan Nephrology Society. Special issue: Clinical practice guidebook for diagnosis and treatment of chronic kidney disease 2012 . Nihon Jinzo Gakkai Shi 54: 1034-1191, 2012 (in Japanese).

11. Lang RM, Badano LP, Mor-Avi V, et al. Recommendations for cardiac chamber quantification by echocardiography in adults: an update from the American society of echocardiography and the European association of cardiovascular imaging. J Am Soc Echocardiogr 28: 1-39, 2015.

12. Klein R, Maisch B, Kochsiek K, Berg PA. Demonstration of organ specific antibodies against heart mitochondria (anti-M7) in sera from patients with some forms of heart diseases. Clin Exp Immunol 58: 283-292, 1984 
13. Otto A, Stähle I, Klein R, Berg PA, Pankuweit S, Brandsch R. Anti-mitochondrial antibodies in patients with dilated cardiomyopathy (anti-M7) are directed against flavoenzymes with covalently bound FAD. Clin Exp Immunol 111: 541-547, 1998.

14. Varga J, Heiman-Patterson T, Muñoz S, Love LA. Myopathy with mitochondrial alterations in patients with primary biliary cirrhosis and antimitochondrial antibodies. Arthritis Rheum 36: 1468-1475, 1993.

15. Berg PA. The role of the innate immune recognition system in the pathogenesis of primary biliary cirrhosis: a conceptual view. Liver Int 31: 920-931, 2011.

16. Pender MP. CD8+ T-cell deficiency, Epstein-Barr virus infection, vitamin D deficiency, and steps to autoimmunity: a unifying hypothesis. Autoimmune Dis 2012: 189096, 2012.

17. Keresztes K, Istenes I, Folhoffer A, et al. Autonomic and sensory nerve dysfunction in primary biliary cirrhosis. World J Gastroen- terol 10: 3039-3043, 2004.

18. Chen PS, Tan AY. Autonomic nerve activity and atrial fibrillation. Heart Rhythm 4: S61-S64, 2007.

19. Beuers U, Gershwin ME, Gish RG, et al. Changing nomenclature for PBC: From 'cirrhosis' to 'cholangitis'. Hepatology 62: 1620$1622,2015$.

20. Working Subgroup (English version) for Clinical Practice Guidelines for Primary Biliary Cirrhosis. Guideline for the management of primary biliary cirrhosis. The Intractable Hepatobiliary Disease Study Group supported by the Ministry of Health, Labour and Welfare of Japan. Hepatol Res 44 (Suppl S1): 71-90, 2014.

The Internal Medicine is an Open Access article distributed under the Creative Commons Attribution-NonCommercial-NoDerivatives 4.0 International License. To view the details of this license, please visit (https://creativecommons.org/licenses/ by-nc-nd/4.0/).

(C) 2017 The Japanese Society of Internal Medicine http://www.naika.or.jp/imonline/index.html 